\title{
Modeling Brown Dwarfs, L Dwarfs, and T Dwarfs
}

\author{
Adam Burrows \\ Department of Astronomy, The University of Arizona, Tucson, AZ, \\ USA 85721
}

\begin{abstract}
In this contribution, I review a subset of our recent efforts in spectral and opacity modeling aimed at guiding the study of brown dwarfs, L dwarfs, and $\mathrm{T}$ dwarfs. I discuss theoretical calculations of the alkali line profiles, newly generated $\mathrm{CrH}$ opacities, new evidence for refractory rainout in $\mathrm{T}$ dwarfs from optical spectral measurements, and the controversy concerning the distinction between brown dwarfs and planets.
\end{abstract}

\section{Introduction}

The subject of brown dwarfs (and substellar-mass objects in general) is entering a new phase of rapid expansion and discovery. More than $200 \mathrm{~L}$ dwarfs are now known, and they are joined by $\sim 30 \mathrm{~T}$ dwarfs. This development requires a corresponding expansion in theoretical effort, involving at its core evolutionary, spectral, and compositional modeling. Crucial to spectral modeling are molecular and atomic opacities, many of which have not been addressed before with the degree of seriousness and completeness that standard stellar atmospheres studies have long enjoyed. However, the pace of relevant spectral and opacity calculations is accelerating and in this spirit I summarize in this paper a few such topics of recent interest. In $\S 2$., I show new proof of the "rainout" and settling of refractory elements in $\mathrm{T}$ dwarfs. Rainout leaves as one of its consequences the lower-temperature reaches of $\mathrm{T}$ dwarf atmospheres enhanced in sodium and potassium atoms. In $\S 3$., I touch on some new calculations of the alkali line wings to many 1000 's of $\AA$ detunings. The red wing of the K I line centered at $0.77 \mu \mathrm{m}$ in particular defines much of the $\mathrm{T}$ dwarf continuum between 0.77 $\mu \mathrm{m}$ and $1.0 \mu \mathrm{m}$. This is followed in $\S 4$. by a short discussion of the new $\mathrm{CrH}$ opacities recently generated by our group. Finally, and a bit tentatively, in $\S 5$. I finish with a short discussion on nomenclature, a subject that continues to exercise the brown dwarf and extrasolar planet communities.

\section{A New Proof of Rainout/Depletion in T Dwarfs}

As explained in Burrows, Marley, and Sharp (2000, BMS), rainout depletes the atmospheres of the refractory elements $\mathrm{Ca}, \mathrm{Al}, \mathrm{Mg}, \mathrm{Fe}$, and $\mathrm{Si}$. In particular, rainout suppresses the formation of alkali feldspars and enables atomic $\mathrm{Na}$ and $\mathrm{K}$ to survive to lower temperatures and pressures, at which point they form $\mathrm{Na}_{2} \mathrm{~S}(\mathrm{c})$ and $\mathrm{KCl}(\mathrm{c})$ (Lodders 1999). As a consequence, their influence on the 
emergent spectrum in the "optical" between $0.5 \mu \mathrm{m}$ and $1.1 \mu \mathrm{m}$ is enhanced. This effect is seen dramatically for the first time in Fig. 1. At $\sim 0.7 \mu \mathrm{m}$, the data for Gliese 570D (Burgasser et al 2001), the coolest known T dwarf, clearly favor the "rainout" model. This fact reiterates the conclusion of BMS that the wings of the $\mathrm{Na} \mathrm{D}$ and $\mathrm{K} \mathrm{I}(7700 \AA)$ resonance lines and aggressive rainout of heavy metals (with the resulting enhancement of the sodium and potassium abundances at altitude) are required to fit the $\mathrm{T}$ dwarf data shortward of 1.0 $\mu \mathrm{m}$. It is shortward of $\sim 1.0 \mu \mathrm{m}$ that the alkali chemistry can be most readily probed (Burrows et al. 2002a).

\section{Alkali Metal Line Profiles}

To continue on the alkali theme, in standard stellar atmospheres, atomic lines are superposed on a background continuum and the concepts of equivalent width and curve of growth make conceptual and practical sense. An individual line is but a perturbation on the local spectrum. However, due to the rainout of metals in cool atmospheres and the consequent paucity of continuum and alternate opacity sources between $0.4 \mu \mathrm{m}$ and $1.0 \mu \mathrm{m}$, the wings of the strong resonance doublets centered at $\sim 7700 \AA$ (K I) and $5890 \AA(\mathrm{Na}-\mathrm{D})$ assume the role of the continuum throughout most of this spectral range (BMS; Tsuji, Ohnaka, and Aoki 1999). In particular, the red wing of the $4 s^{2} S_{1 / 2}-4 p^{2} P_{3 / 2}$ transitions of $\mathrm{K}$ I provides the pseudo-continuum in cool molecular atmospheres all the way from $0.77 \mu \mathrm{m}$ to $\sim 1.0 \mu \mathrm{m}$. The Na-D doublet, centered as it is in the middle of the visible, determines the true color of brown dwarfs (magenta/purple; Burrows et al. 2001).

Hence, whereas in traditional stellar atmospheres the Lorentzian core and Gaussian wings of a line are not generally of relevance beyond $\sim 20 \AA$ detunings ( $\Delta \lambda$ from the line core), in cool substellar atmospheres the relevant reach of the $\mathrm{Na} I$ and K I resonance lines can be thousands of $\AA$. Given this, to achieve accurate spectral fits for brown dwarf, L dwarf, $\mathrm{T}$ dwarf, and hot giant planet atmospheres, the shapes of the far wings of these alkali lines as a function of pressure and temperature must be ascertained.

Recently, Burrows and Volobuyev (2002c) performed ab initio calculations of the energy shifts of the ground and excited states of sodium and potassium immersed in $\mathrm{H}_{2}$ - and helium-rich atmospheres and have obtained the opacity profiles of the red and blue wings of the $\mathrm{Na}-\mathrm{D}$ and $\mathrm{K}$ I resonance lines using the Unified Franck-Condon formalism (Szudy and Baylis 1975,1996). The absorption spectra as a function of photon wavelength for the $\mathrm{D}_{2}$ and $\mathrm{D}_{1}$ lines of $\mathrm{Na}$ and $\mathrm{K}$ centered near $5890 \AA$ and $7700 \AA$, respectively, are depicted in Fig. 2 at a temperature of $1000 \mathrm{~K}$ and a total pressure of one atmosphere. The contributions at various orientation angles of $\mathrm{H}_{2}$, the angle-integrated spectra (solid), and the results for the $\mathrm{Na}+\mathrm{He}$ and $\mathrm{K}+\mathrm{He}$ systems are shown. The Lorentzian cores are not included on this plot.

As seen in Fig. 2, the cutoff on the red wing of the potassium feature due to an exponential term containing the ground-state interaction potential of the $\mathrm{K}+\mathrm{H}_{2}$ system is seen to be situated between $0.95 \mu \mathrm{m}$ and $1.0 \mu \mathrm{m}$, close to the $0.98 \mu \mathrm{m}$ used in Burrows et al. (2002c). The cutoff of the red wing of the $\mathrm{Na}$ feature is near $0.8 \mu \mathrm{m}$, but is a bit more gradual. The corresponding cutoffs for 


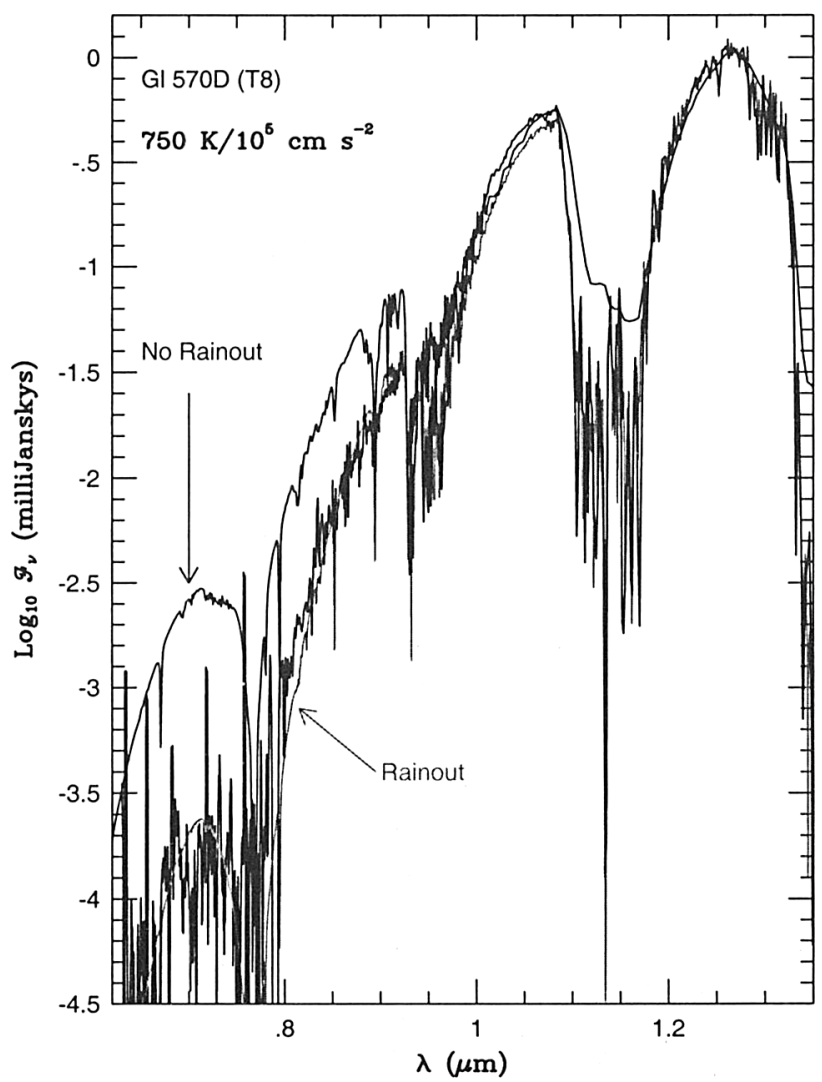

Figure 1. A comparison of the measured absolute flux $\left(\mathrm{F}_{\nu}\right)$, in milliJanskys) of Gliese 570D (thick, black) with two solar-metallicity model spectra for wavelengths $(\lambda)$ from $0.6 \mu \mathrm{m}$ to $1.4 \mu \mathrm{m}$. The best-fit line incorporates the rainout of silicates and the resulting persistence of $\mathrm{Na}$ and $\mathrm{K}$ at altitude, while the upper line is for strict chemical equilibrium without rainout. From the significant $(\times 10)$ difference between the data and the "No rainout" model near $0.7 \mu \mathrm{m}$, we see evidence for the role of rainout in $\mathrm{T}$ dwarf atmospheres. The Gliese 570D models assume $\mathrm{T} /$ gravity $=\left[750 \mathrm{~K} / 10^{5} \mathrm{~cm} \mathrm{~s}^{-2}\right]$. 


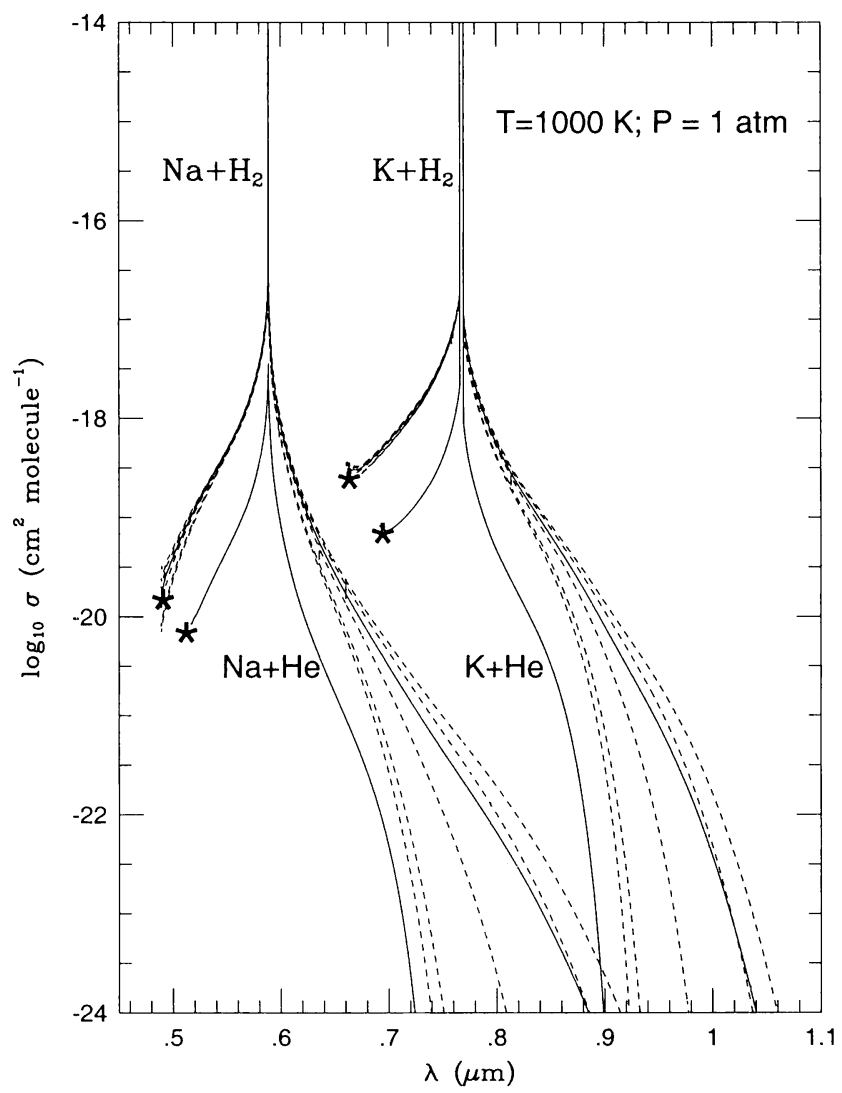

Figure 2. Absorption cross sections (in $\mathrm{cm}^{2}$ ) versus wavelength (in $\mu \mathrm{m}$ ) for the Na-D and K I doublets at $5890 \AA$ and $7700 \AA$, respectively, using the quasi-static theory of wing line profiles. The Lorentzian line cores are not included on this plot. Cross sections are shown for the different orientations of the $\mathrm{H}_{2}$ molecule $\left(\theta=0^{\circ}, 20^{\circ}, 45^{\circ}, 70^{\circ}, 90^{\circ}\right)$, as well as after integrating over angle (solid). The behavior is monotonic with angle, from steepest (small angle) to shallowest (large angle). Also depicted are the cross sections due to perturbations by the spherical helium atom (with $\mathrm{C}_{\infty}$ symmetry). The asterisks indicate the positions of the rainbow satellites on the blue wings. A temperature of $1000 \mathrm{~K}$ and a total pressure of one atmosphere have been used. The partial pressure of $\mathrm{He}$ is assumed to be $\sim 10 \%$ of this total (Anders and Grevesse 1989). 
the alkali-He systems are more abrupt, but of less importance due to the lower abundance of helium. Importantly, unlike in the algorithm of BMS, there are no free parameters for this theory of line profiles; the cutoff on the red wing of the K I feature appears naturally. Fig. 2 also makes clear that the red and blue wings are asymmetrical.

\section{New CrH Opacities}

The 1-0 and 0-0 bands of the $\mathrm{A}^{6} \Sigma^{+}-\mathrm{X}^{6} \Sigma^{+}$transition of $\mathrm{CrH}$ are used as primary markers for the new L dwarf spectral class (Kirkpatrick et al. 1999a,b). Hence, accurate $\mathrm{CrH}$ line lists and oscillator strengths are needed to calculate the $\mathrm{CrH}$ opacities now used to model the spectral energy distributions of these transitional and substellar objects.

Burrows et al. (2002b) have recently created a program for generating $\mathrm{CrH}$ cross sections for the six band sequences with band heads from $\sim 0.7 \mu \mathrm{m}$ to $\sim$ $1.4 \mu \mathrm{m}$. Figure 3 depicts the resulting $\mathrm{CrH}$ cross sections for representative pressures and temperatures at which $\mathrm{CrH}$ is typically found in substellar atmospheres. Specifically, opacity spectra for $T / P$ pairs of $1500 \mathrm{~K} / 10$ bars, 2000 $\mathrm{K} / 10$ bars, and $2000 \mathrm{~K} / 100$ bars are portrayed. The corresponding opacities using the older database for the $0-0$ transition of $\mathrm{CrH}$ of Ram, Jarman, and Bernath (1993), as calculated by $R$. Freedman (private communication), are two orders of magnitude weaker. The lion's share of the difference between the old and the new CrH opacities can be traced to an increase by a factor of $\sim 13.5$ in the oscillator strength and to a previously inappropriate division by 6 , the electronic spin degeneracy factor. The $\mathrm{A}-\mathrm{X} \quad 0-0$ band at $\sim 0.86$ microns (just shortward of the neighboring $\mathrm{FeH}$ feature at $\sim 0.87$ microns) and the $\mathrm{A}-\mathrm{X} 0-1$ $\mathrm{CrH}$ band near 0.997 microns (just longward of the classic Wing-Ford band of $\mathrm{FeH}$ ) are prominent and, in principle, diagnostic features in measured L dwarf spectra (Kirkpatrick et al. 1999a,b).

Since $\mathrm{CrH}$ is a defining molecule of the $\mathrm{L}$ dwarf spectroscopic class, accurate opacities as a function of temperature and pressure are necessary for spectral syntheses and to extract $\mathrm{CrH}$ abundances for $\mathrm{L}$ dwarf atmospheres. Burrows et al. (2002b) have used the new theoretical opacity data to obtain an abundance for the L5 dwarf 2MASSI J1507038-151648 (Kirkpatrick et al. 1999b). Figure 4 depicts this comparison. The $\mathrm{CrH} / \mathrm{H}_{2}$ number ratio they find is $\sim 2-4 \times 10^{-9}$, in reasonable agreement with expectations.

\section{What's in a Name?}

The deuterium burning cut was used originally by some to distinguish planets from brown dwarfs. This was partly because of a figure in Burrows et al. (1997) that arbitrarily distinguished evolutionary trajectories in luminosity/age space on this basis, calling one class that burned tens of percent or more of their deuterium brown dwarfs and the others that did not "planets" (as I recall, the original figure had the quotes as well). Also, one has the unfounded prejudice that a planet should not have a thermonuclear phase.

One should distinguish giant planets from brown dwarfs by their mode of formation, by their origin. Since an object's origin is not easily "detected" or 


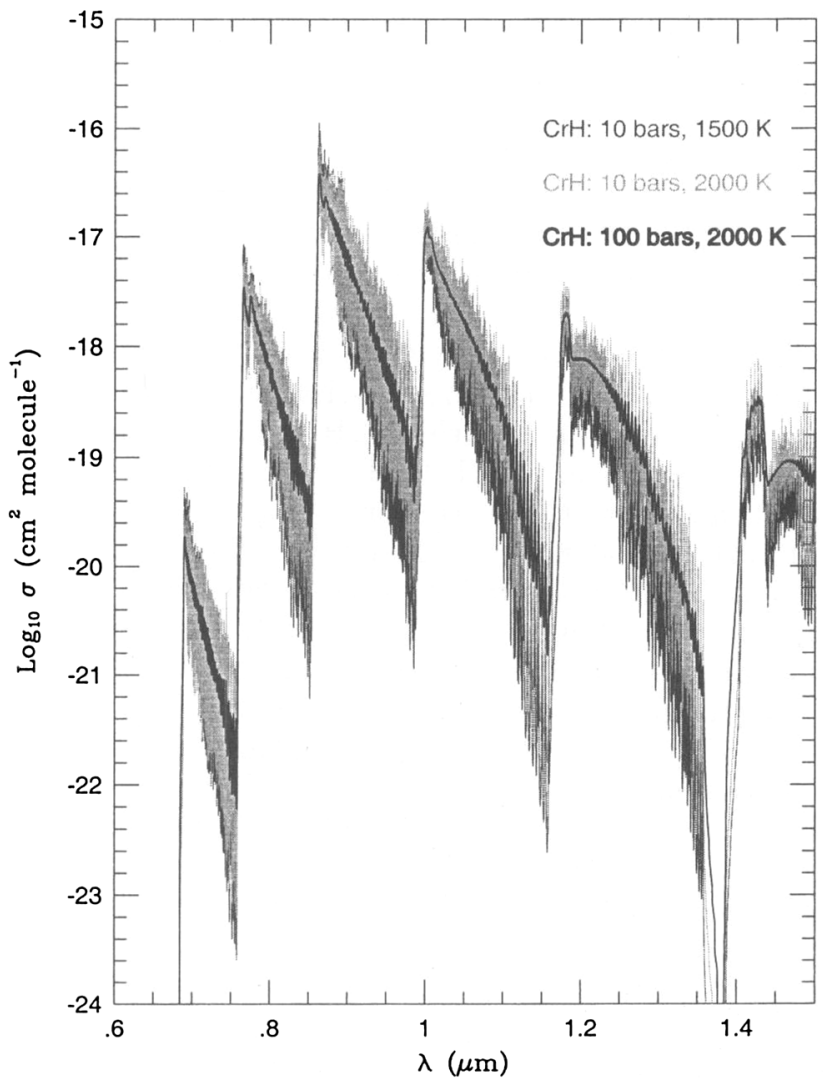

Figure 3. The logarithm (base ten) of the absorption cross section of $\mathrm{CrH}$ versus wavelength (in microns) from $\sim 0.7 \mu \mathrm{m}$ to $1.5 \mu \mathrm{m}$, at various temperatures and pressures. The narrow (central) curve at 100 bars and $2000 \mathrm{~K}$ depicts the effect of large pressure broadening (when compared with the other curves at 10 bars). A comparison of the slightly upper curve $(10 \mathrm{bars}, 2000 \mathrm{~K})$ with the lower curve $(10$ bars, $1500 \mathrm{~K}$ ) portrays the effect of increasing temperature. The A-X $0-0$ band is the strongest and is the third from the left near $\sim 0.9 \mu \mathrm{m}$. As the temperature and pressure decrease the cross section range in a given band widens and manifests larger variation with wavelength. 


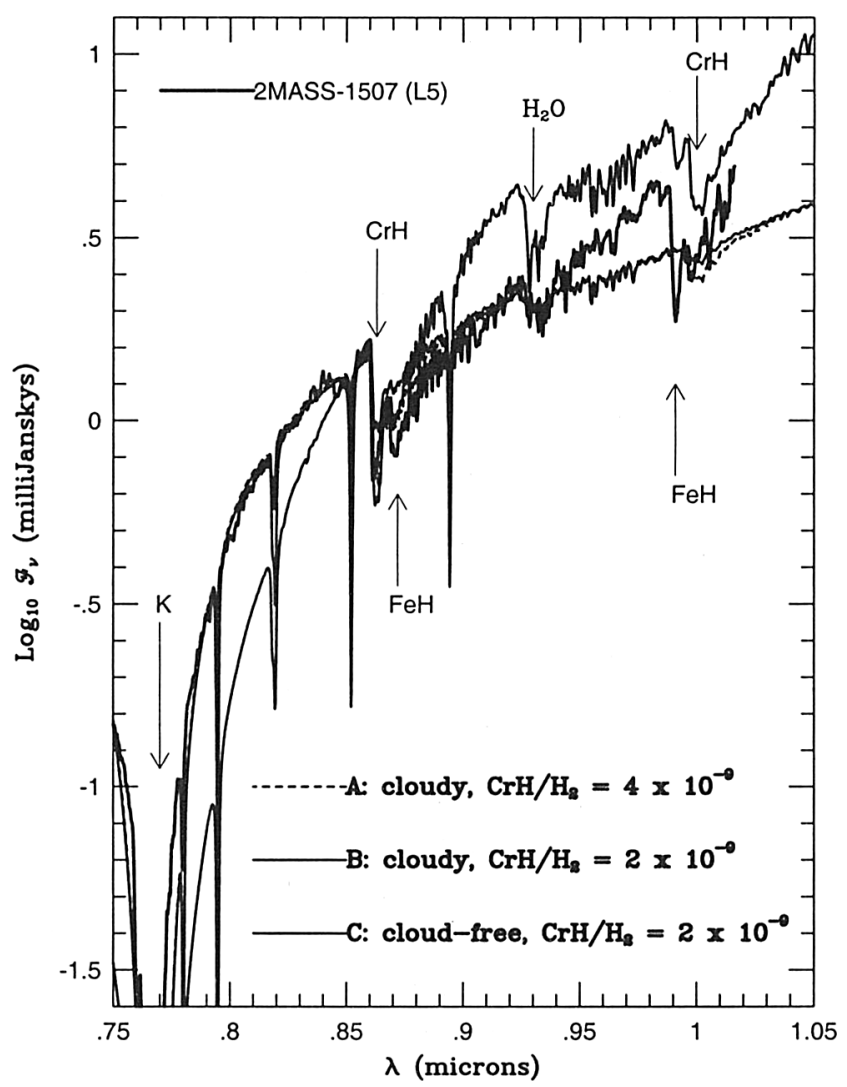

Figure 4. The $\log$ (base ten) of the absolute flux density $\left(\mathcal{F}_{\nu}\right)$ in milliJanskys versus wavelength $(\lambda)$ in microns from $0.75 \mu \mathrm{m}$ to 1.05 $\mu \mathrm{m}$ for self-consistent theoretical solar-metallicity models of the L5 dwarf 2MASS-1507. Also included are the corresponding data (thick) for 2MASS-1507 from Kirkpatrick et al. (1999b). All models are for $\mathrm{T}_{\text {eff }}=1700 \mathrm{~K}$ and a gravity of $10^{5.5} \mathrm{~cm} \mathrm{~s}^{-2}$. The dashed line depicts a model (A) with a forsterite cloud and a $\mathrm{CrH} / \mathrm{H}_{2}$ number abundance ratio of $4 \times 10^{-9}$, the solid line (almost on top of the dashed line, except near $\mathrm{CrH}$ features) depicts a model (B) with a forsterite cloud and a $\mathrm{CrH} / \mathrm{H}_{2}$ number abundance ratio of $2 \times 10^{-9}$, and the steeper line depicts a cloud-free model $(\mathrm{C})$ with a $\mathrm{CrH} / \mathrm{H}_{2}$ number abundance ratio of $2 \times 10^{-9}$. Below temperatures of $1400 \mathrm{~K}$, the $\mathrm{CrH}$ abundance was set to zero. Indicated with arrows are the positions of the $\mathrm{CrH}, \mathrm{FeH}, \mathrm{H}_{2} \mathrm{O}$, and $\mathrm{K} \mathrm{I}(7700 \AA)$ features in this spectral range. Also prominent are the Cs I lines at $8523 \AA$ and $8946 \AA$, the Na I line at $8195 \AA$, and the $\mathrm{Rb}$ I lines at $7802 \AA$ and $7949 \AA$. These spectra have been deresolved to an $R(\lambda / \Delta \lambda)$ of 1000 . 
discerned, the situation is ready-made for confusion. In fact, there is really no need to define and codify the nomenclature nor the name to be applied to any particular object or class of objects at this time; people just want (perhaps a bit too rigidly and with unjustified Procrustean zeal) to name things, or to stuff objects into cubbyholes.

There is ambiguity in the provenance of these objects and this ambiguity will be with us for a while. If one uses a particular term (planet or brown dwarf), one should always include the definition one is employing. Not to do so implies that a standard, acceptable definition has been arrived at; despite what some have written and the IAU, such is not the case.

Hence, formation history is a key, but not yet known. One would expect a planet (or most of them) to be orbiting a star (or brown dwarf). There may be metallicity (composition) differences between objects with the same mass, but different origins or modes of formation. Eccentricity is probably not a good discriminant (though this is not known). Very few "planets" are expected to be flung out of their cradles. Brown dwarfs are just the tail of the stellar mass distribution, but the mass distributions of brown dwarfs and giant planets may overlap.

When classifying a newly-discovered substellar-mass object, one can use a variety of "reasonable" criteria, but the tentative classification is just that, tentative. The usefulness of a classification scheme at this stage in the development of the two related subjects of brown dwarfs and giant planets is inversely proportional to its rigidity. A flexible and open-minded philosophy towards nomenclature is best, which more data and information will progressively guide towards a more and more reasonable naming scheme. It may be that the mass distributions of planets and brown dwarfs don't overlap, that their compositions will enable one to distinguish one from the other, etc. Until then, I prefer a bit of ambiguity to the illusion of clarity and the pretense of certainty. When there is fog, to acknowledge its presence is more honest than to try to ignore it.

Acknowledgments. The author thanks Bill Hubbard, Jonathan Lunine, David Sudarsky, Ivan Hubeny, Christopher Sharp, Drew Milsom, Maxim Volobuyev, Curtis Cooper, and Jonathan Fortney for informative exchanges and collaboration, as well as NASA for its financial support via grants NAG5-10760 and NAG5-10629.

\section{References}

Anders, E. and Grevesse, N. 1989, Geochim. Cosmochim. Acta, 53, 197

Burgasser, A.J., et al. 2002, ApJ, 564, 421

Burrows, A., Marley M., Hubbard, W.B. Lunine, J.I., Guillot, T., Saumon, D. Freedman, R., Sudarsky, D. and Sharp, C.M. 1997, ApJ, 491, 856

Burrows, A., Marley, M.S., and Sharp, C.M. 2000, ApJ, 531, 438

Burrows, A., Hubbard, W.B., Lunine, J.I., and Liebert, J. 2001, Rev. Mod. Phys., 73, 71

Burrows, A., Burgasser, A.J., Kirkpatrick, J.D., Liebert, J., Milsom, J.A., Sudarsky, D., and Hubeny, I. 2002a, ApJ,, 573, 394 
Burrows, Ram, R, Bernath, P, Sharp, C., and Milsom, J.A. 2002b, ApJ, in press Burrows, A. and Volobuyev, M. 2002c, submitted to ApJ

Kirkpatrick, J.D., Reid, I.N., Liebert, J., Cutri, R.M., Nelson, B., Beichman, C.A., Dahn, C.C., Monet, D.G., Gizis, J., and Skrutskie, M.F. 1999a, ApJ, 519, 802

Kirkpatrick, J.D., Allard, F., Bida, T., Zuckerman, B., Becklin, E.E. Chabrier, G., Baraffe, I. 1999b, ApJ, 519, 834

Lodders, K. 1999, ApJ, 519, 793

Ram, R.S., Jarman, C.N. and Bernath, P.F. 1993, J. Mol. Spectrosc., 161, 445

Szudy, J. and Baylis, W. 1975, J. Quant. Spectrosc. Radiat. Transfer, 15, 641

Szudy, J. and Baylis, W. 1996, Physics Reports, 266, 127

Tsuji, T., Ohnaka, K., and Aoki, W. 1999, ApJ, 520, L119 


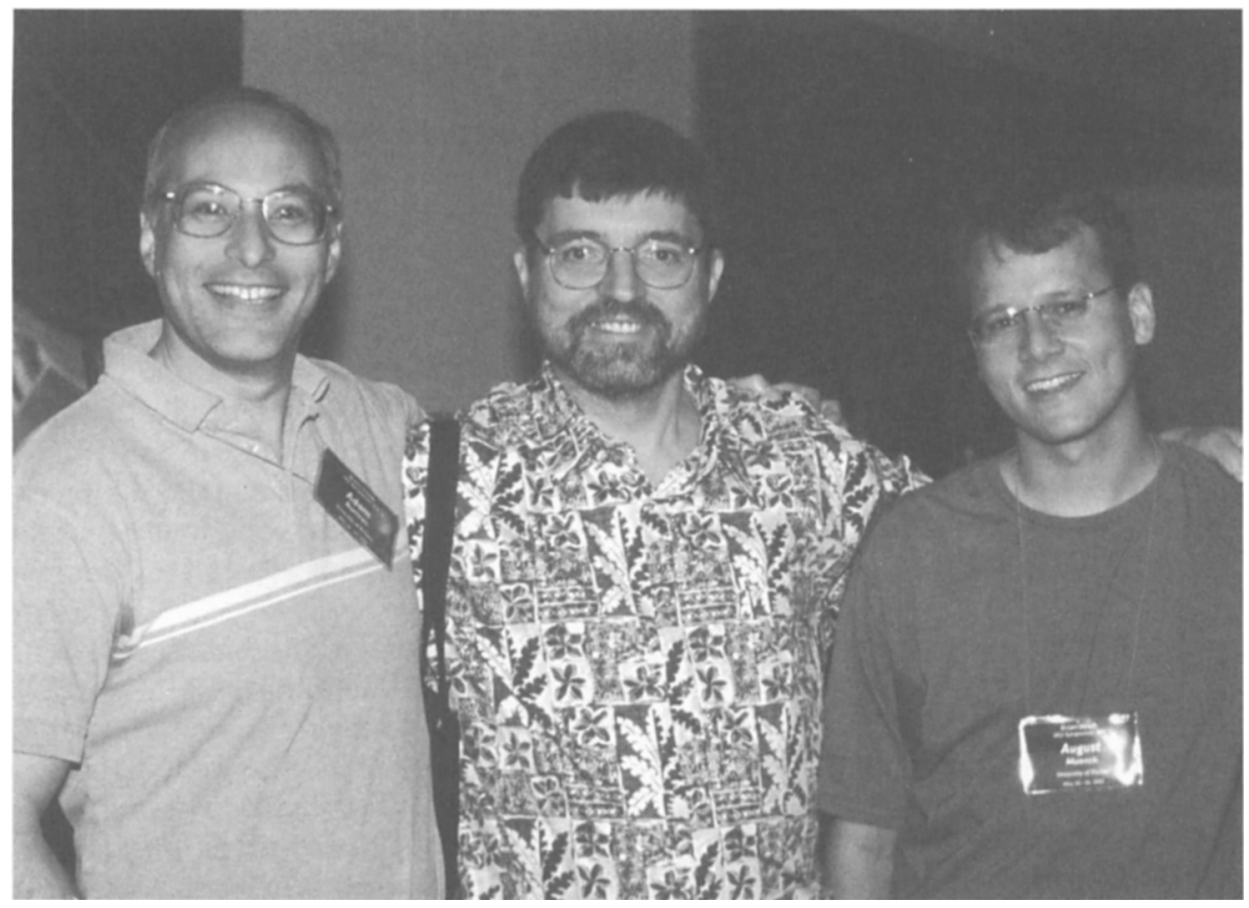

From left to right: Adam Burrows, Charlie Lada, and August Muench 